\title{
A Study on Bacteriological Profile of Skin Infections and Antimicrobial Susceptibility of Causative Organisms among Children in a Tertiary Care Hospital
}

\author{
J. Pandian and R. Vikram Balaji* \\ Government Thiruvannamalai Medical College, Tamil Nadu, India \\ *Corresponding author
}

\begin{abstract}
A B S T R A C T
Pyoderma is the commonest skin infection in children. The awareness about regional

Keywords

Pyoderma,

Antimicrobial

resistance, Children

Article Info

Accepted:

16 February 2018

Available Online:

10 March 2018 pattern of isolates, their antibiotic sensitivity and resistance pattern is of utmost importance in treating them. Aims of the study are 1) To study the bacteriological profile of pyoderma in children 2) To assess the antibiotic sensitivity pattern. 3) To determine the resistance pattern. Cases of primary and secondary pyoderma in paediatric age group attending Dermatology outpatient department in a tertiary care teaching hospital over a period of one year were enrolled in this study. Swabs were taken, identification test, antimicrobial sensitivity tests to rule out resistant strains were done. Primary pyoderma $72.5 \%$ was the noted, Staphylococcus aureus was the commonest isolate $76 \%$ followed by Streptococcus pyogenes 7\%, both MSSA and MRSA were 100\% sensitive to Vancomycin, Linezolid and Mupirocin, Streptococcus were $100 \%$ sensitive to Penicillin. This study shows the present trend of the isolates among pyoderma in paediatric age group, the antimicrobial susceptibility and resistant pattern of the isolates. So that a correct antibiotic policy could be formulated by making culture and sensitivity mandatory for all pyodermal lesions.
\end{abstract}

\section{Introduction}

The most important innate immune organ in our body is skin; it protects our body by blocking the UV rays that affects the epidermal and dermal layer; it constantly do vigilance by immune surveillance with the help of Langerhan cells and acidic environment; Skin of the infantile and pediatric age group are more prone for infections because of increased exposure to trauma and allergies (Chen et al., 2008). Previous studies have estimated that at least $30 \%$ of outpatient clinics have dermatology diseases in pediatric age group (Hayden, 1985; Jawade et al., 2015). Skin diseases can be either of infective, autoimmune or hereditary type; Infective causes are numerous and a collective term named as Pyoderma is used to refer the infection of skin layers and its adnexal structures namely hair follicle, sebaceous gland, apocrine and eccrine glands; Pyoderma includes many skin infections that are caused by bacterial agents mostly by Staphylococcus aureus and group A $\beta$ hemolytic Streptococci (Sladden and Johnston, 2004). Usually skin infections in children are treated in OPD with any 
appropriate antimicrobial susceptibility testing that in turn leads to increased resistance profile. Methicillin resistant Staphylococcus aureus are increasingly prevalent in all infections especially from skin and soft tissues infections making it more vulnerable to get transmitted to infant and pediatric age group (Pate et al., 2014). Hence there is a need to estimate the bacteriological profile and its corresponding antimicrobial susceptibility pattern to start proper empirical treatment in severe cases and to avoid spreading resistance patterns;

This study was aimed to isolate the bacterial organisms that are associated with pyoderma (skin infections) in children under age group of 12 years and to study the antimicrobial susceptibility pattern of each isolates.

\section{Materials and Methods}

This prospective study was conducted over a period of one year in a tertiary care teaching hospital from samples obtained in dermatology OPD and processed in Department of microbiology.

Total samples collected from children under age group of 12 were about 200. Children who had already received a course of antimicrobials were excluded from the study.

\section{Sample collection}

Skin lesions were visualised and looked for whether papulated or crusted or necrosed lesions; The area was cleaned with $70 \%$ alcohol solution with proper precautions; the crust or necrosed tissues were removed slowly and with a sterile forceps the flap was removed; with the help of two sterile cotton swabs, the area was rolled and sample collected. In case of pustules, the swab for rolled from centre to periphery in a circular manner, with the help of a sterile needle the pustule was ruptured and the let out pus was collected in a sterile swab.

\section{Sample processing}

A single swab was utilised for Gram staining by direct smear preparation. Gram stained smear was visualised under microscope and viewed for pus cells and organisms. Another swab was utilised for bacterial culture by plating the swab successively in nutrient agar, blood agar and Mac Conkey agar. The plates were incubated aerobically at $35-37^{\circ} \mathrm{C}$ for 24 hours. Growth was then identified by colony morphology, Gram staining and then by following biochemical reactions. Series of tests that were done are Catalase test, Modified Oxidase test, Methyl red, VogesProskauer tests, Indole test, Bacitracin susceptibility test, Urease test, Slide and Tube coagulase test, Bile esculin agar test, Growth at 10 and $45^{\circ} \mathrm{C}$ for Enterococci, PYR test and Sugar fermentation test.

\section{Antimicrobial susceptibility testing}

The colonies that were confirmed as organism were then subjected to antimicrobial susceptibility testing (AST) according to CLSI guidelines. Around three to five colonies of same morphological type were selected for antibiogram. They were inoculated in $4-5 \mathrm{ml}$ of nutrient broth. And incubated at $35-37^{\circ}$ until the inoculum matches 0.5 McFarland standard. Mueller Hinton agar was used as a basal media for AST and for fastidious organisms - MHA with 5\% sheep blood agar was used. Antimicrobial discs (HiMedia) were used as five discs per plate;

Antibiotic discs used for Staphylococcus aureus were Cotrimoxazole, Penicillin, Gentamycin, Amoxicillin, Erthromycin, Azithromycin, Clindamycin, Cephalexin, Cefoxitin, Doxycycline, Ciprofloxacin, Linezolid, Teicoplanin and Mupirocin; 
Antibiotic discs used for Streptococcus pyogenes were Bacitracin, Cotrimoxazole, Penicillin, Erythromycin, Clindamycin, Doxycycline, Linezolid, Vancomycin, Ciprofloxacin, Ceftriaxone and Teicoplanin; The antimicrobial groups used for Enterococcus group were Ampicillin, Erthromycin, Ciprofloxacin, Doxycycline, Linezolid and Vancomycin. Zone of inhibition was measured and interpreted according to CLSI guidelines.

\section{Results and Discussion}

Over a period of one year, 200 samples were subjected to study and following results were obtained; In our study, children less than 1 month of age were $10.5 \%(\mathrm{n}=21)$, 1month1year $24.5 \%(\mathrm{n}=49)$, 1year to 5years $44 \%$ $(\mathrm{n}=88)$ and $5-12$ years group $21 \% \quad(\mathrm{n}=42)$. Most of the children in preschool group were victims of pyodermal lesions. Prevalence of pyoderma was more in male children $57 \%$ $(\mathrm{n}=114)$, than female children $43 \% \quad(\mathrm{n}=86)$ which constitutes a ratio of 1.3:1.

Lesions were classified clinically as Primary pyoderma and Secondary pyoderma. Impetigo, folliculitis, carbuncle, furuncle, Ecthyma, Erysipelas, Paronychia, Cellulitis comes under primary pyoderma. Traumatic lesions, burns, eczematous dermatitis, scabies with secondary infection, chronic ulcers, intertrigo, Sebaceous cyst, pilonidal cyst, pyodermal gangrenosa, hidradenitis suppurativa and vesicular eruptions comes under secondary pyoderma. (Bowen et al., 2015) The pattern of Primary pyoderma observed in our centre shows Impetigo as the single largest group which accounts for $53.1 \%(\mathrm{n}=77)$ cases. Followed by folliculitis with $20 \% \quad(\mathrm{n}=30 \%)$ and furunculosis and Ecthyma each 9. 6\%, Cellulitis 3. 4\% ( $\mathrm{n}=5)$, Paronychia 2\% ( $\mathrm{n}=3)$ and Omphalitis $1 \% \quad(\mathrm{n}=2)$. Secondary pyoderma constituted $27.5 \%$ among the isolates. The common lesion observed in them were Scabies $45 \%(\mathrm{n}=25)$, insect bite $34.5 \%$ $(n=19)$, contact dermatitis $10.9 \%(n=6)$ with secondary infection, ulcer $3.6 \% \quad(\mathrm{n}=2)$ followed by one case $1.8 \% \quad(n=1)$ each of burns, pediculosis and intertrigo.

Bacteriological culture showed culture positivity in $91.5 \%(\mathrm{n}=181)$ and no growth in 9.5\% $(\mathrm{n}=19)$ of the samples. Among the culture positive cases, Staphylococcus aureus was isolated as single largest group accounting for $76 \%(\mathrm{n}=152)$, Streptococcus pyogenes in $7 \%(\mathrm{n}=14)$ Enterococcus faecalis in 1\% $(\mathrm{n}=2)$ of cases. Mixed organisms (Staphylococcus aureus with Streptococcus pyogenes) were observed in $6.5 \%(\mathrm{n}=13)$ cases (Table 1$)$.

Among the Staphlococcus aureus isolates penicillin was least sensitive $1 \%$, Cotrimoxazole 48\%, Gentamicin 63.1\%, Amoxicillin 57.2\%, Erythromycin 70\%, Ciprofloxacin 72\%, Clindamycin 80\%, Cephalexin 78.9\%, Azithromycin 82\%, Cefoxitin 92\%, Doxycycline $97 \%$ and Linezolid, Vancomycin, Teicoplanin, Mupirocin all showed $100 \%$ sensitive. Staphylococcus aureus from polymicrobial cases showed $100 \%$ cefoxitin sensitivity. Streptococcus pyogenes were sensitive to Erythromycin 71.5\%, Gentamycin 78.5\%, Ciprofloxacin $85.7 \%$ and Bacitracin, Penicillin, Doxycyclline, Linezolid, Vancomycin, Ceftriaxone, Clindamycin all were $100 \%$ sensitive. Two Enterococcus faecalis isolates in our study. $50 \%$ of the isolates were sensitive to Ampicillin and Erythromycin. Ciprofloxacin, Doxycycline, Linezolid, Vancomycin, Teicoplanin and High level Gentamycin were 100\% sensitive (Table 2). Skin infections (Pyoderma) are the most common problem followed by Respiratory infections in pediatric age group. Cutaneous Staphylococcal and Streptococcal infections are more common in this age group and may lead to cellulitis and septicemia. 
Table.1 Distribution of causative agent associated with pyoderma

\begin{tabular}{|c|c|c|c|c|c|}
\hline Pyoderma & Clinical type & S.aureus & S.pyogenes & Enterococci & Polymicrobial \\
\hline \multirow{7}{*}{$\begin{array}{l}\text { Primary } \\
\text { pyoderma }\end{array}$} & Impetigo & 56 & 7 & - & 7 \\
\hline & Folliculitis & 29 & 2 & - & - \\
\hline & Ecthyma & 7 & 4 & - & 1 \\
\hline & Furuculosis & 11 & - & - & - \\
\hline & Omphalitis & 2 & - & - & - \\
\hline & Paronychia & 3 & - & - & - \\
\hline & Cellulitis & 3 & 1 & 1 & - \\
\hline \multirow{6}{*}{$\begin{array}{l}\text { Secondary } \\
\text { pyoderma }\end{array}$} & Scabies with secondary infection & 19 & - & 1 & 1 \\
\hline & Insect bite with secondary infection & 15 & - & - & 2 \\
\hline & Contact dermatitis with infection & 4 & - & - & 1 \\
\hline & Ulcer with infection & 1 & - & - & 1 \\
\hline & Burns with infection & 1 & - & - & - \\
\hline & Pediculosis with secondary infection & 1 & - & - & - \\
\hline TOTAL & & 152 & 14 & 2 & 13 \\
\hline
\end{tabular}

Table.2 Antimicrobial susceptibility pattern of clinical isolated obtained from pyoderma

\begin{tabular}{|c|c|c|c|c|c|c|c|}
\hline \multirow[t]{2}{*}{ S. No } & \multirow{2}{*}{$\begin{array}{l}\text { Name of the } \\
\text { Antibiotic }\end{array}$} & \multicolumn{2}{|c|}{ S. aureus $(\mathrm{n}=152)$} & \multicolumn{2}{|c|}{ S. pyogenes $(\mathrm{n}=14)$} & \multicolumn{2}{|c|}{ E. faecalis $(n=2)$} \\
\hline & & $\mathbf{S}$ & $\%$ & $\mathbf{S}$ & $\%$ & $\mathbf{S}$ & $\%$ \\
\hline 1 & Penicillin (10 units) & 2 & 1 & 14 & $100 \%$ & - & - \\
\hline 2 & Cotrimoxazole $(25 \mu \mathrm{g})$ & 73 & $48 \%$ & - & - & - & - \\
\hline 3 & Gentamicin $(10 \mu \mathrm{g})$ & 96 & $63.1 \%$ & 11 & $78.5 \%$ & - & - \\
\hline 4 & Amoxicillin $(30 \mu \mathrm{g})$ & 87 & $57.2 \%$ & - & - & - & - \\
\hline 5 & Erythromycin $(15 \mu \mathrm{g})$ & 107 & $70 \%$ & 10 & $71.5 \%$ & 1 & $50 \%$ \\
\hline 6 & Ciprofloxacin $(5 \mu \mathrm{g})$ & 109 & $72 \%$ & 12 & $85.7 \%$ & 2 & $100 \%$ \\
\hline 7 & Clindamycin $(2 \mu \mathrm{g})$ & 121 & $80 \%$ & 14 & $100 \%$ & - & - \\
\hline 8 & Doxycycline $(30 \mu \mathrm{g})$ & 148 & $97 \%$ & 14 & $100 \%$ & 2 & $100 \%$ \\
\hline 9 & Cephalexin $(30 \mu \mathrm{g})$ & 120 & $78.9 \%$ & - & - & - & - \\
\hline 10 & Cefoxitin $(30 \mu \mathrm{g})$ & 140 & $92 \%$ & - & - & - & - \\
\hline 11 & Azithromycin $(15 \mu \mathrm{g})$ & 126 & $82 \%$ & - & - & - & - \\
\hline 12 & Linezolid $(30 \mu \mathrm{g})$ & 152 & $100 \%$ & 14 & $100 \%$ & 2 & $100 \%$ \\
\hline 13 & Vancomycin $(30 \mu \mathrm{g})$ & 152 & $100 \%$ & 14 & $100 \%$ & 2 & $100 \%$ \\
\hline 14 & Teicoplanin $(30 \mu \mathrm{g})$ & 152 & $100 \%$ & - & - & 2 & $100 \%$ \\
\hline 15 & Mupirocin $(200 \mu \mathrm{g})$ & 152 & $100 \%$ & - & - & - & - \\
\hline 16 & Bacitracin $(0.04 \mathrm{U})$ & - & - & 14 & $100 \%$ & - & - \\
\hline 17 & Ampicillin $(10 \mu \mathrm{g})$ & - & - & - & - & 1 & $50 \%$ \\
\hline 18 & Ceftriaxone $(30 \mu \mathrm{g})$ & - & - & 14 & $100 \%$ & - & - \\
\hline 19 & High level Gentamycin $(120 \mu \mathrm{g})$ & - & - & - & - & 2 & $100 \%$ \\
\hline
\end{tabular}


(Sladden and Johnston, 2004) The above study was conducted to estimate the burden of skin infections in childhood and to analyse the antimicrobial susceptibility pattern. There was a male preponderance of $57 \%$ infections in above study group with female children $43 \%$. This is similar to that of other study conducted near to the locality of above study and may be due to more exposure for male children during playing and other activities (Jose, n.d.; Poudyal et al., 2016). Distribution of cases based on age group showed that maximum number of cases were in preschool age of about 1-5 years compared to school going age group. This observation may be due to infections following minor trauma that occurs in preschool children (Bowen et al., 2015; Jawade et al., 2015; Vamseedhar1 et al., 2015). Impetigo was the predominant skin disease among all infections. Increased prevalence of malnutrition, insect bites, lack of awareness about skin protection are the factors making more preponderance for impetigo (Bowen et al., 2015; Pereira, 2014). Infections secondary to another cause was studied in detail and it showed scabies with secondary infection was the most common among them (Johnston and Sladden, 2005; Tyring et al., 2016).

Distribution of causative organisms showed Staphylococcus aureus (76\%) as the major isolate followed by Streptococcus pyogenes (7\%) and Enterococcus faecalis (1\%). Most of the skin infections in children and adults also are caused by Staphylococcus aureus. They cause variety of presentations like carbuncle, furuncle, abscess, impetigo and cellulitis. Rising resistance strains like community acquired MRSA awakens the treating clinician to use appropriate local or systemic antimicrobials (Farley, 2008; Hinckley and Allen, 2008). Nasal carriage of MRSA strains among the vulnerable population who are in contact with the children or children themselves are the source of resistant skin infections (Creech et al., 2015). Antimicrobial susceptibility of S.aureus strains showed increased resistance to penicillin, MRSA strains (identified by Cefoxitin disk diffusion method) as $7.9 \%$ and $100 \%$ sensitive to vancomycin and higher group of antimicrobials. When compared to other studies which states that MRSA prevalence in India is around $40 \%$ and most commonly isolated in skin and soft tissue infections (Joshi et al., 2013). MRSA prevalence is lesser in the above study was only $7.9 \%$, mainly because of the paediatric age group. (Hamdy et al., 2017; Yonezawa et al., 2015)

Streptococcus pyogenes is responsible for skin infections like erysipelas, impetigo, cellulitis and necrotising fasciitis. (Stevens and Bryant, 2016) In the above study $7 \%$ of total infections are caused by Streptococcus pyogenes. Most of the studies have shown that S.pyogenes is the second most common cause following $S$. aureus in causing skin and soft tissue infections especially in pediatric age group. (Bowen et al., 2015; Chiller et al., 2001; Stevens and Bryant, 2016) Antimicrobial susceptibility pattern of S. pyogenes implied that $100 \%$ sensitivity was seen with Penicillin, Ceftriaxone and Vancomycin. Some degree of resistance was seen with Gentamicin, Erythromycin and Ciprofloxacin. This coincides with other studies also where there is no proven Penicillin resistance in $S$. pyogenes. Treating $S$. pyogenes skin infections in children is utmost important in order to prevent sequalae like post streptococcal glomerulonephritis (Dillon, 1970; Ikebe et al., 2005; RodriguezIturbe and Haas, 2016) .

Enterococcus faecalisis a rising cause for most of the nosocomial infections especially secondary skin and soft tissue infections (Agudelo Higuita and Huycke, 2014). According to previous study, incidence of 
Enterococcal skin infections was 8.6 per 1000 admissions in New Delhi and among them $E$. faecalis was 3.4 per 1000 admissions and $E$. faecium was 4.8 per 1,000 admissions (Rajkumari et al., 2014). The increased incidence leads to increased antimicrobial resistance also. Enterococci has an intrinsic resistance to several antibiotics (Patel et al., 2013). Hence Vancomycin which is often given for severe infections also leads to rising vancomycin resistance (Brandl et al., 2008; Humphreys, 2014). In the above study vancomycin was susceptible in all the isolates. This pattern is mainly due to decreased sample isolation with Enterococci and samples were only skin and soft tissues. But, overall the third most common cause is Enterococcal skin infections in children and hence antimicrobial usage should be restricted according to CLSI guidelines.

In most instances, treating clinicians prefer empirical treatment for skin and soft tissue infections. Multidrug resistance has become a clinical challenge. Our study clearly shows the change in trend of isolates and rising antibiotic resistance pattern among them. In this context clinicians should be aware of varied presentation of resistant strains and should implement strict antimicrobial testing for all the samples before starting higher group of antimicrobials.

\section{References}

Agudelo Higuita, N.I., Huycke, M.M., 2014. Enterococcal Disease, Epidemiology, and Implications for Treatment, in: Gilmore, M.S., Clewell, D.B., Ike, Y., Shankar, N. (Eds.), Enterococci: From Commensals to Leading Causes of Drug Resistant Infection. Massachusetts Eye and Ear Infirmary, Boston.

Bowen, A.C., Mahé, A., Hay, R.J., Andrews, R.M., Steer, A.C., Tong, S.Y.C., Carapetis, J.R., 2015. The Global
Epidemiology of Impetigo: A Systematic Review of the Population Prevalence of Impetigo and Pyoderma. PloS One 10, e0136789. https://doi. org/10.1371/journal.pone.0136789

Brandl, K., Plitas, G., Mihu, C.N., Ubeda, C., Jia, T., Fleisher, M., Schnabl, B., DeMatteo, R.P., Pamer, E.G., 2008. Vancomycin-resistant Enterococci exploit antibiotic-induced innate immune deficits. Nature 455, 804-807. https://doi.org/10.1038/nature07250.

Chen, G.-Y., Cheng, Y.-W., Wang, C.-Y., Hsu, T.-J., Hsu, M.M.-L., Yang, P.-T., Chen, W.-C., 2008. Prevalence of skin diseases among schoolchildren in Magong, Penghu, Taiwan: a community-based clinical survey. J. Formos. Med. Assoc. Taiwan Yi Zhi 107, 21-29. https://doi.org/10.1016/ S0929-6646 (08) 60004-2.

Chiller, K., Selkin, B.A., Murakawa, G.J., 2001. Skin Microflora and Bacterial Infections of the Skin. J. Investig. Dermatol. Symp. Proc. 6, 170-174. https://doi.org/10.1046/j.0022-202x.200 1.00043.x.

Creech, C.B., Al-Zubeidi, D.N., Fritz, S.A., 2015. Prevention of Recurrent Staphylococcal Skin Infections. Infect. Dis. Clin. North Am. 29, 429-464. https://doi.org/10.1016/j.idc.2015.05.00 7.

Dillon, H.C., 1970. The treatment of streptococcal skin infections. J. Pediatr. 76, 676-684. https://doi.org/10.1016/ S0022-3476 (70) 80284-0

Farley, J.E., 2008. Epidemiology, clinical manifestations, and treatment options for skin and soft tissue infection caused by community-acquired methicillinresistant Staphylococcus aureus. J. Am. Acad. Nurse Pract. 20, 85-92. https://doi.org/10.1111/j.1745-7599.20 07.00290.x. 
Hamdy, R.F., Hsu, A.J., Stockmann, C., Olson, J.A., Bryan, M., Hersh, A.L., Tamma, P.D., Gerber, J.S., 2017. Epidemiology of Methicillin-Resistant Staphylococcus aureus Bacteremia in Children. Pediatrics e20170183. https://doi.org/10.1542/peds.2017-0183

Hayden, G.F., 1985. Skin diseases encountered in a pediatric clinic. A oneyear prospective study. Am. J. Dis. Child. 1960 139, 36-38.

Hinckley, J., Allen, P.J., 2008. Communityassociated MRSA in the pediatric primary care setting. Pediatr. Nurs. 34, 64-71.

Humphreys, H., 2014. Controlling the spread of vancomycin-resistant Enterococci. Is active screening worthwhile? J. Hosp. Infect. 88, 191-198. https://doi.org/10. 1016/j.jhin.2014.09.002

Ikebe, T., Hirasawa, K., Suzuki, R., Isobe, J., Tanaka, D., Katsukawa, C., Kawahara, R., Tomita, M., Ogata, K., Endoh, M., Okuno, R., Watanabe, H., Japan, the W.G. for G.A.S. in, 2005. Antimicrobial Susceptibility Survey of Streptococcus pyogenes Isolated in Japan from Patients with Severe Invasive Group A Streptococcal Infections. Antimicrob. Agents Chemother. 49, 788-790. https://doi.org/10.1128/AAC.49.2.788790.2005

Jawade, S.A., Chugh, V.S., Gohil, S.K., Mistry, A.S., Umrigar, D.D., 2015. A Clinico-Etiological Study of Dermatoses in Pediatric Age Group in Tertiary Health Care Center in South Gujarat Region. Indian J. Dermatol. 60, 635. https://doi.org/10.4103/0019-5154. 169147

Johnston, G., Sladden, M., 2005. Scabies: diagnosis and treatment. BMJ 331, 619622.

Jose, n.d. Prevalence of common dermatoses in school children of rural areas of
Salem; a region of South India [WWW Document].

Joshi, S., Ray, P., Manchanda, V., Bajaj, J., Chitnis, D.S., Gautam, V., Goswami, P., Gupta, V., Harish, B.N., Kagal, A., Kapil, A., Rao, R., Rodrigues, C., Sardana, R., Devi, K.S., Sharma, A., Balaji, V., 2013. Methicillin resistant Staphylococcus aureus (MRSA) in India: Prevalence \& susceptibility pattern. Indian J. Med. Res. 137, 363369.

Pate, A.J., Terribilini, R.G., Ghobadi, F., Azhir, A., Barber, A., Pearson, J.M., Kalantari, H., Hassen, G.W., 2014. Antibiotics for methicillin-resistant Staphylococcus aureus skin and soft tissue infections: the challenge of outpatient therapy. Am. J. Emerg. Med. 32, 135-138. https://doi.org/10.1016/j. ajem.2013.10.026

Patel, G., Snydman, D.R., the AST Infectious Diseases Community of Practice, 2013. Vancomycin-Resistant Enterococcus Infections in Solid Organ Transplantation. Am. J. Transplant. 13, 59-67. https://doi.org/10.1111/ajt.12099

Pereira, L.B., 2014. Impetigo - review. An. Bras. Dermatol. 89, 293-299. https:// doi.org/10.1590/abd18064841.20142283

Poudyal, Y., Ranjit, A., Pathak, S., Chaudhary, N., 2016. Pattern of Pediatric Dermatoses in a Tertiary Care Hospital of Western Nepal. Dermatol. Res. Pract. 2016, 6306404. https://doi.org/10.1155/2016/6306404

Rajkumari, N., Mathur, P., Misra, M.C., 2014. Soft Tissue and Wound Infections Due to Enterococcus spp. Among Hospitalized Trauma Patients in a Developing Country. J. Glob. Infect. Dis. 6, 189-193. https://doi.org/ 10.4103/0974-777X.145253

Rodriguez-Iturbe, B., Haas, M., 2016. PostStreptococcal Glomerulonephritis, in: 
Ferretti, J.J., Stevens, D.L., Fischetti, V.A. (Eds.), Streptococcus pyogenes: Basic Biology to Clinical Manifestations. University of Oklahoma Health Sciences Center, Oklahoma City (OK).

Sladden, M.J., Johnston, G.A., 2004. Common skin infections in children. BMJ 329, 95-99.

Stevens, D.L., Bryant, A.E., 2016. Impetigo, Erysipelas and Cellulitis, in: Ferretti, J.J., Stevens, D.L., Fischetti, V.A. (Eds.), Streptococcus pyogenes: Basic Biology to Clinical Manifestations. University of Oklahoma Health Sciences Center, Oklahoma City (OK).
Tyring, S.K., Lupi, O., Hengge, U.R., 2016. Tropical Dermatology E-Book. Elsevier Health Sciences.

Vamseedhar, Y., Ahamed, S.M., Kumar, U.V., Mohan4, C.R., 2015. Skin diseases, Children, Pyoderma, Scabies. STUDY PATTERN SKIN Dis. Child. Present. RIMS KADAPA P.

Yonezawa, R., Kuwana, T., Kawamura, K., Inamo, Y., 2015. Invasive CommunityAcquired Methicillin-Resistant Staphylo coccus aureus in a Japanese Girl with Disseminating Multiple Organ Infection: A Case Report and Review of Japanese Pediatric Cases [WWW Document]. Case Rep. Pediatr. https://doi.org/10.1155/2015/291025

\section{How to cite this article:}

Pandian, J. and Vikram Balaji, R. 2018. A Study on Bacteriological Profile of Skin Infections and Antimicrobial Susceptibility of Causative Organisms among Children in a Tertiary Care Hospital. Int.J.Curr.Microbiol.App.Sci. 7(03): 1827-1834. doi: https://doi.org/10.20546/ijcmas.2018.703.215 